\title{
Decreasing CCT deviation of white light emitting diodes by employing $\mathrm{SiO}_{2}$ nanoparticles
}

\author{
My Hanh Nguyen Thi ${ }^{1}$, Phung Ton That ${ }^{2}$ \\ ${ }^{1}$ Faculty of Mechanical Engineering, Industrial University of Ho Chi Minh City, Viet Nam \\ ${ }^{2}$ Faculty of Electronics Technology, Industrial University of Ho Chi Minh City, Ho Chi Minh city, Vietnam
}

\begin{abstract}
Article Info
Article history:

Received Dec 15, 2020

Revised Mar 17, 2021

Accepted Apr 1, 2021

Keywords:

CCT deviation

Luminous flux

Mie-scattering theory

ABSTRACT

In this research, the $\mathrm{SiO}_{2}$ nano-particles (NPs) usage in enhancing optical performances of InGaN/GaN-based white light-emitting diodes (WLEDs) with remote phosphor structure. The research subject shows better lighting capacity than the white LEDs devices without the space between the layers. The adjustment in development process resulted in enhancements of internal quantum efficiency (IQE) and light extraction efficiency (LEE) that lead to $13.5 \%$ luminous efficacy improvement. From the experiments, it can be concluded that the LEE is affected by the trapped light and enhancing the light output with $\mathrm{SiO}_{2}$ scattering properties reduce the amount of trapped light. These results confirm that $\mathrm{SiO}_{2}$ nano-particles is effective in enhancing the optical performance of WLEDs and can be considered for production of higher quality devices.
\end{abstract}

$\mathrm{SiO}_{2}$ nano-particles

This is an open access article under the CC BY-SA license.

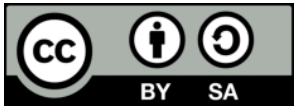

\section{Corresponding Author:}

Phung Ton That

Faculty of Electronics Technology

Industrial University of Ho Chi Minh City

No. 12 Nguyen Van Bao Street, Ho Chi Minh City, Vietnam

Email: tonthatphung@iuh.edu.vn

\section{INTRODUCTION}

As the growing demands of lighting applications to meet the higher living standard, it requires more efficient illuminating materials. Light emitting diodes (LEDs) and laser diodes are widely used materials in light emitting devices since they can provide high optical quality, however, there are still some aspects that can be improved for higher result. The lattice-mismatch causing high threading dislocation (TD) density of $\mathrm{GaN}$ developed on foreign substrates is one of the critical issues [1], [2]. In the traditional GaN on sapphire substrate the TD density can be up to $10^{8}-10^{10} \mathrm{~cm}^{2}$ [3]. Epitaxial lateral overgrowth (ELO), a promising technique that manages to prevent the dislocation from being propagated, has been extensively studied as a solution to achive lower dislocation density and higher the internal quantum efficiency (IQE) [4]. Besdies, the light extraction efficiency (LEE) in GaN-based LEDs is degraded by the total internal reflection (TIR), which occurs at a low angle and leads to discrepancy of refractive index between air and GaN. As a result, the light transmission from the multi quantum well (MQW) of LEDs to surrounding environment is quite low [5]. To enhance LEE, structures with air gaps attached to GaN-based LEDs were proposed and have shown positive results in IQE and external quantum efficiency (EQE) [6]-[8]. IQE in InGaN/GaN blue LEDs has received many improvements that are close to be $100 \%$, therefore, it is more effective for EQE studies to research the solution LEE enhancement [9]. The epoxy or silicone encapsulating have the quality that is suitable for serial production with high cost-effectiveness, simple process. The encapsulation that covers the outside of $\mathrm{GaN}$ devices can reduce the refractive index discrepancy at the air-GaN interface and also enhance the LEE [8]. The patterned structure was also proposed to enhance the LEE by reducing the total internal 
reflection [10]. The issue with this solution is that the encapsulation loses the effect from roughening when being utilized after texturing. Therefore, both encapsulation and texturing will cancel each other, which makes LEE enhancement from texturing greatly reduced if it is followed by the encapsulation application [11], [12]. From these reactions, the LEE and EQE by using both texturing and encapsulating should not be applied. Instead of applying to the outer side of LED, the texturing can be put inside LED layers. The reason for this solution is to prevent the later surface-encapsulation modifying the pattern on textured surface, thus making the simultaneous application of texturing and encapsulating become possible. If the two solutions are successfully combined, the LED package with GaN growth on the patterned sapphire substrate (PSS) can present $60 \%$ increase in lumen performance [13]. In particular, the slant patterns' surfaces in the PSS divert TDs sideways and thus, decreasing TDs density directed at the surface, and boost the IQE [14]. Moreover, these PSS patterns' slanted sides expand the angle of radiation transmission and lower the impact of TIR. Accordingly, the PSS shows broader cone angle of photon transmission to the substrate and photon reflection, compared to the non-patterned sapphire substrate. This also happens to the photons yielded from the lower side of MQW active region, leading to benefited LEE [15]. Considering these positive results, we arrange a structure that apply the PSS in designing an epitaxial structure for greater performance.

Based on previous studies, it can be concluded that the air gaps with hollow three-sided prism and cone shapes on the conventional substrate are essential to get the desired epitaxial structure that can result in better LEE [16]. The air-void formation is carried out with the initial deposition of $\mathrm{SiO}_{2}$ nanoparticle layer on the traditional substrate, followed by the process of patterning, ELO and wet etching. So, if the substrate is PSS, the air-void and the PSS-surface patterns share the same position. However, it is difficult to get a uniform ELO due to the differences in the size and form of the air-void and the PSS patterns. Another solution that can be named is to use a film of hollow silica nanospheres for the reduction in TD density and pressure and the improvement in LEE [17]. This method carried out with the growth of silica nanospheres on the non-patterned sapphire substrate and the nano-scale ELO. Similar to the former, this combination did not attain the uniformity of ELO as the surface patterns of PSS and the silica nanospheres did not compatible in their dimension and shape. From these results, it can be seen that directly combining PSS with the air-voids or silica nanospheres developed on traditional substrate did not work as expected. This implies that the separation between the adjusted elements of the epitaxial structure and the surface of the PSS is essential to get the former to enhance not only crystalline quality but also scattered lights. Therefore, a LED structure with GaN nano-pillars was introduced to put this idea into practice and achieve the enhanced lighting efficiency [18], [19]. Besides from the modification of $\mathrm{SiO}_{2}$ concentration, the refractive index was also changed to match the amount of $\mathrm{SiO}_{2}$. The refractive index for silicone is 1.4 , phosphor is 1.8 , and $\mathrm{SiO} 2$ particles is 2.23. As a result, the total refractive index of the mentioned components must be calculated with the following equation: $\mathrm{RI}=\mathrm{V}_{1} \mathrm{RI}_{1}+\mathrm{V}_{2} \mathrm{RI}_{2}+\mathrm{V}_{3} \mathrm{RI}_{3}$. In this equation, $\mathrm{V}_{1}, \mathrm{~V}_{2}$ and $\mathrm{V}_{3}$ are the mass percentages of the materials in the layer. In lighting configuration with $\mathrm{SiO}_{2}$ particles, the $\mathrm{SiO}_{2}$ was doped at the ratio of $1 \%$ to $3 \%$ of the phosphor material in the configuration. As a result, the refractive indices of the phosphor material in each layer were 1.428 and 1.445 [20]. The TFCalc32 simulation was employed as a tool to analyze the impacts of various refractive index in the layers. The result demonstrated that the extracted light from $\mathrm{SiO}_{2}$ configuration is almost identical to the amount obtained in the conventional configuration. This is due to the similar refractive index in these configurations are similar. This suggests that the light output improvement achieved in $\mathrm{SiO}_{2}$ configuration mostly comes from the scattering properties of $\mathrm{SiO}_{2}$. To accurately evaluate the impact of $\mathrm{SiO}_{2}$ particles, we applied the Mie-scattering theory to examine the scattering results of $\mathrm{SiO}_{2}$ nanoparticles at various concentrations. A simplified model with only the $\mathrm{SiO}_{2}$ nanoparticles and silicone gel in the material layers were applied in the calculation to reduce the complexity and increase accuracy. The particle diameter is $300 \mathrm{~nm}$ and the $\mathrm{SiO}_{2}$ scattering events were calculated at $1 \%$ and $3 \% \mathrm{SiO}_{2}$. The measured result of refractive index at $460 \mathrm{~nm}$ for this configuration was 2.23 , the scattering intensity was able to hit $100 \%$ at $500 \mathrm{~nm}$ and began to drop at wavelength higher than $500 \mathrm{~nm}$. The simulated outcomes and the testing results regarding the scattering efficiency were compatible. In the wavelength range from $300-700 \mathrm{~nm}$, the scattering intensity was quite similar in lighting configuration with high amount of $\mathrm{SiO}_{2}[21]$.

The innovative LED structure with GaN nano-pillars located on the PSS and combining with $\mathrm{SiO}_{2}$ nano-particles (NPs) is proposed to prevent the propagation of dislocations along the vertical direction of the air-void growth on sapphire substrate. The results shows that this LED structure can increase the lighting efficacy up to approximately $300 \%$. The light output enhancement can be push even further by filling $\mathrm{SiO}_{2}$ into the air-void among the nano-pillars [3]. As mentioned above, LED-surface texturing cannot effectively work to heighten the LEE as the subsequently encapsulating will degraded the LEE improvement of roughened surface. Therefore, the enhancement of lumen output recorded from the experiments could be considered to be yielded from inside the device. 


\section{PREPARATION AND COMPUTATION}

\subsection{Preparation of $\mathrm{SiO}_{2}$}

The optical properties of $\mathrm{SiO}_{2}$ NPs embedded PSS LEDs used in the experiment were evaluated with reference to traditional PSS LEDs (without $\mathrm{SiO}_{2} \mathrm{NPs}$ embedded nano-pillars). The process of $\mathrm{SiO}_{2}-$ doped InGaN/GaN LED developed on PSS is depicted in Figure 1. Before doping the silica nano-particles, the undoped $\mathrm{GaN}$ layer ( $\mathrm{u}-\mathrm{GaN})$ with the particle size of $3 \mu \mathrm{m}$ was deposited on the PSS using metalorganic chemical vapor deposition (MOCVD) method. Then, to create the nano-pillars in this GaN layer, a layer of $\mathrm{SiO}_{2} \mathrm{NPs}$ with a thickness of $100 \mathrm{~nm}$ was placed on the surface of the $\mathrm{u}-\mathrm{GaN}$ via plasma-enhanced chemical vapor deposition. Subsequently, a Ni film having 10-nm thickness was put on the $\mathrm{SiO}_{2}$ layer by e-beam evaporation. Next, the process of rapid thermal annealing (RTA) was carried out to this 3-layer sample of $\mathrm{Ni}$, $\mathrm{SiO}_{2}$ and $\mathrm{u}-\mathrm{GaN}$ to attain the $\mathrm{Ni}$ dot mask on top of the $\mathrm{SiO}_{2}$ layer, which is essential for the next etching process. The annealing process was operated with $\mathrm{N}_{2}$ flow and at the temperature of $850^{\circ} \mathrm{C}$ for about a minute. The silica nano-particle layer was then patterned with an etching technique of inductively coupled plasma (ICP) under $\mathrm{O}_{2}$ and $\mathrm{CF}_{4}$ gases. Afterwards, the etching process was continued with the gas flow of $\mathrm{Cl}_{2}$ to cut down the u-GaN thickness to $1.5 \mu \mathrm{m}$ [22]. The buffered oxide etchant was subsequently utilized to remove the remaining $\mathrm{Ni}$ dots on the top surface of the nano-pillars of the $\mathrm{GaN}$ template. $\mathrm{SiO}_{2} \mathrm{NPs}_{\text {, having }}$ particle size of $100 \mathrm{~nm}$, were added into the air-void on the GaN nano-pillar template by using 5000-rpm spin coating for 30 seconds. The GaN nano-pillar with embedded $\mathrm{SiO}_{2} \mathrm{NPs}$ was then applied for ELO process. A layer of $\mathrm{GaN}$ was regrown on the top of the nano-pillars, and after they became firmly attached, the layers of Si-doped n-GaN, InGaN/GaN multi quantum wells, and Mg-embedded p-GaN were placed, relatively. It is noted that the thickness of Si-doped n-GaN and Mg-embedded p-GaN layers are $3 \mu \mathrm{m}$ and $200 \mathrm{~nm}$, in turn. Simultaneously, the reference structure of undoped GaN grown on PSS was packed for comparison.

The field emission scanning electron microscope was applied used to capture the cross-section of the $\mathrm{SiO}_{2}$-embedded GaN-based LED. Photoluminescence mapping measurements were conducted at room temperature, with the excitation source of helium-cadmium (He-Cd) laser using the $325 \mathrm{~nm} \mathrm{UV}$ line, and having output power of $25 \mathrm{~mW}$. The current-voltage (I-V) and light output power (L-I) characteristics were examined via using on-wafer probing with indium contacts. The comparison between the results of the $\mathrm{SiO}_{2-}$ doped GaN nano-pillars on PSS and the reference GaN non-pillars template were demonstrated to evaluate the effectiveness of the simulated package. It is noted that the fabrication requirements for all types are similar, the only different is that in the modified GaN LED structure, the air-void on the nano-pillar template is filled with $\mathrm{SiO}_{2} \mathrm{NPs}$. The enhanced performance concluded from the experiment is in comparison with the outcome from simulation. The target of using modified GaN structure and PSS is to enhance LEE by applying different setups; thus, the simulation is the tool to understand the mechanism and enable the fullest potential, which means different configurations can still support and complement each other even when the functions are overlapped.

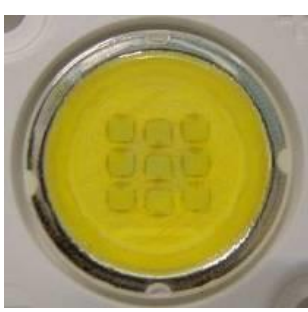

(a)

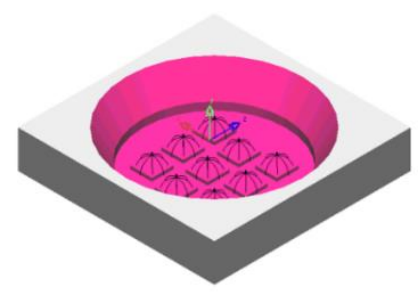

(b)

Figure 1. These figures are, (a) Photograph of WLEDs sample, (b) The simulated WLEDs model

\subsection{Scattering computation}

With Mie-scattering theory [23]-[26], the calculations of the scattering coefficient $\mu_{s c a}(\lambda)$, anisotropy factor $g(\lambda)$, and reduced scattering coefficient $\delta_{\text {sca }}(\lambda)$ are conducted:

$$
\begin{aligned}
& \mu_{s c a}(\lambda)=\int N(r) C_{s c a}(\lambda, r) d r \\
& g(\lambda)=2 \pi \iint_{-1}^{1} p(\theta, \lambda, r) f(r) \cos \theta d \cos \theta d r
\end{aligned}
$$




$$
\delta_{s c a}=\mu_{s c a}(1-g)
$$

$N(r)\left(\mathrm{mm}^{3}\right)$ and $r(\mu \mathrm{m})$ indicate the distribution density and radius of diffusive particles, respectively. $C_{s c a}$ shows the scattering cross sections $\left(\mathrm{mm}^{2}\right), p(\theta, \lambda, r)$ means the phase function, $\lambda(\mathrm{nm})$ is the light wavelength, $\theta$ is the scattering angle $\left({ }^{\circ} \mathrm{C}\right)$, and $f(r)$ is the size distribution function of the diffusive particles in the phosphorous layer. The computation of $f(r)$ can be expressed as:

$$
\begin{aligned}
f(r) & =f_{\text {dif }}(r)+f_{\text {phos }}(r) \\
N(r) & =N_{\text {dif }}(r)+N_{\text {phos }}(r) \\
& =K_{N} \cdot\left[f_{\text {dif }}(r)+f_{\text {phos }}(r)\right]
\end{aligned}
$$

As can be observed from the above equations, $N(r)$ consists of the diffusive particle density $N_{d i f}(r)$ and the phosphor particle density $N_{\text {phos }}(r) . f_{d i f}(r)$ and $f_{\text {phos }}(r)$ present the size distribution function data of the diffusive and phosphorus particles. $K_{N}$ indicates the number of the diffusor unit for one diffusor concentration and can be computed via:

$$
c=K_{N} \int M(r) d r
$$

Where $\mathrm{M}(\mathrm{r})$ is the mass distribution of the diffusive unit, proposed by equation:

$$
M(r)=\frac{4}{3} \pi r^{3}\left[\rho_{\text {dif }} f_{\text {dif }}(r)+\rho_{\text {phos }} f_{\text {phos }}(r)\right]
$$

$\rho_{\text {diff }}(r)$ and $\rho_{\text {phos }}(r)$ show the density of diffusive particles and phosphor grains. Based on Mie theory, $C_{s c a}$ can be reckoned by:

$$
C_{s c a}=\frac{2 \pi}{k^{2}} \sum_{0}^{\infty}(2 n-1)\left(\left|a_{n}\right|^{2}+\left|b_{n}\right|^{2}\right)
$$

where $k=2 \pi / \lambda$, and the mathematic expressions of $a_{n}$ and $b_{n}$ are:

$$
\begin{aligned}
& a_{n}(x, m)=\frac{\psi_{n}^{\prime}(m x) \psi_{n}(x)-\mathrm{m} \psi_{n}(m x) \psi_{n}^{\prime}(x)}{\psi_{n}^{\prime}(m x) \xi_{n}(x)-\mathrm{m} \psi_{n}(m x) \xi_{n}^{\prime}(x)} \\
& b_{n}(x, m)=\frac{m \psi_{n}^{\prime}(m x) \psi_{n}(x)-\psi_{n}(m x) \psi_{n}^{\prime}(x)}{m \psi_{n}^{\prime}(m x) \xi_{n}(x)-\psi_{n}(m x) \xi_{n}^{\prime}(x)}
\end{aligned}
$$

Where $x=k . r, m$ indicates the index of refraction, while $\psi_{n}(x)$ and $\xi_{n}(x)$ display the Riccati-Bessel function. Therefore, the relative refractive indices of diffusive and phosphor particles, $\left(\mathrm{m}_{\mathrm{dif}}\right)$ and $\left(\mathrm{m}_{\mathrm{phos}}\right)$, in the silicone can be computed by using $m_{d i f}=n_{\text {dif }} / n_{\text {sil }}$ and $m_{\text {phos }}=n_{\text {phos }} / n_{\text {sil }}$; then, the phase function can be:

$$
p(\theta, \lambda, r)=\frac{4 \pi \beta(\theta, \lambda, r)}{k^{2} C_{s c a}(\lambda, r)}
$$

where $\beta(\theta, \lambda, r), S_{1}(\theta)$ and $S_{2}(\theta)$ present the angular scattering amplitudes that can be computed by:

$$
\begin{aligned}
& \beta(\theta, \lambda, r)=\frac{1}{2}\left[\left|S_{1}(\theta)\right|^{2}+\left|S_{2}(\theta)\right|^{2}\right] \\
& S_{1}=\sum_{n=1}^{\infty} \frac{2 n+1}{n(n+1)}\left[\begin{array}{l}
a_{n}(x, m) \pi_{n}(\cos \theta) \\
+b_{n}(x, m) \tau_{n}(\cos \theta)
\end{array}\right]
\end{aligned}
$$




$$
S_{2}=\sum_{n=1}^{\infty} \frac{2 n+1}{n(n+1)}\left[\begin{array}{l}
a_{n}(x, m) \tau_{n}(\cos \theta) \\
+b_{n}(x, m) \pi_{n}(\cos \theta)
\end{array}\right]
$$

Where,

- $N(r)$ is the number of diffusional particles (per cubic millimeter), or diffusional density distribution

- $\quad C_{s c a}$ is the scattering cross-section (in square millimeters)

$-\lambda$ is the wavelength of the incident light (in nanometers)

$-\quad r$ is the radius of each particle (in micrometers)

$-\theta$ is the scattering angle (degrees)

$-p(\theta, \lambda, r)$ is the phase function

- $f(r)$ is the size distribution function of $\mathrm{ZnO}$ in the phosphor layer

$-g(\lambda)$ is the anisotropy factor

- $x$ is the size parameter

- $m$ is the refractive index

- $a_{n}$ and $b_{n}$ are the expansion coefficient with even and odd symmetries, in turn

$-\pi_{\mathrm{n}}(\cos \theta)$ and $\tau_{\mathrm{n}}(\cos \theta)$ are the angular dependent functions.

An encapsulating process with $\mathrm{SiO}_{2}$ for $\mathrm{CdSe}-\mathrm{ZnS}$ core-shell QDs in hydrophobic solvent was carried out. The purpose of this 2-stage process is to fabricate $\mathrm{SiO}_{2}$ nanocomposites with high QD concentration. The self-assembled QDs were prepared using spray-drying technique. This technique is used to evaporate the solvent and get the QDs in each droplet to be self-assembled by capillary force [17]. Particularly, the QDs mixed into toluene solvent were ultrasonically sprayed at the temperature of $250^{\circ} \mathrm{C}$. During the spraying, the QDs aggregated to form complex-shaped porous clusters. These clusters were then uniformly blended into the hydrophilic ethanol. It is noted that the hydrophobic properties of self-assembled QDs are maintained after the spray-drying process. In the next stage, the tetraethyl orthosilicate (TEOS) and aqueous ammonia solution $\left(\mathrm{NH}_{4} \mathrm{OH}\right)$ were added to encapsulated assembled QD clusters with silicananocomposites. After a two-hour hydrolysis of TEOS and $\mathrm{NH}_{4} \mathrm{OH}, \mathrm{SiO}_{2}$ nano-films were directly coated the QDs, and the $\mathrm{SiO}_{2}$-ecapsulated QD has a size of approximate $15 \mathrm{~nm}$. Then, the hydrolytic reaction was continued to increase the particle size of $\mathrm{QD}-\mathrm{SiO}_{2}$ nanocomposites to a few micrometers, see Figure 2.

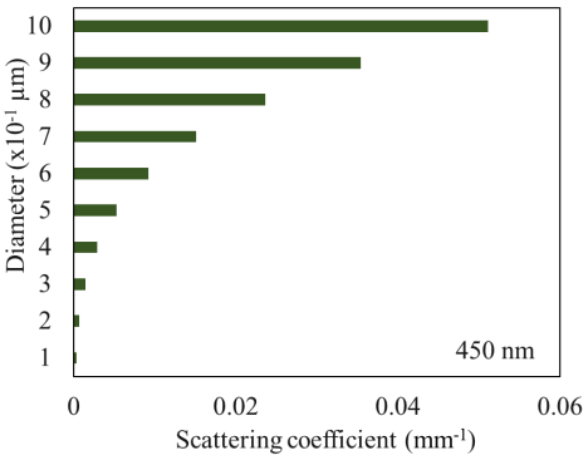

(a)

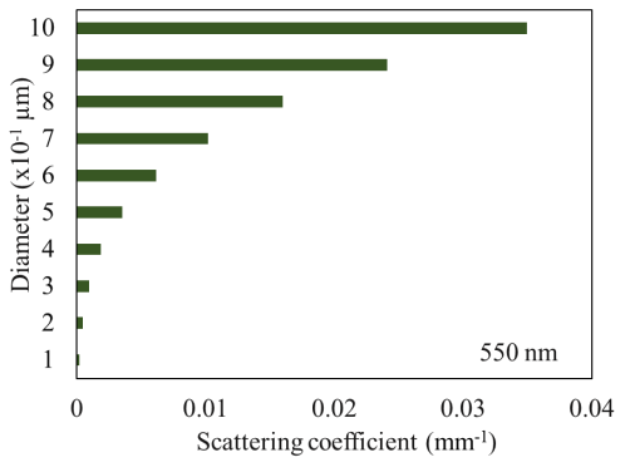

(b)

Figure 2. Scattering coefficients of $\mathrm{SiO}_{2}$ particles at, (a) $450 \mathrm{~nm}$, (b) $550 \mathrm{~nm}$

Accordingly, the photoluminescence (PL) spectra changing during the time of $\mathrm{SiO}_{2}$ encapsulating were observed and displayed in Figure 2. It showed a high degradation in the $\mathrm{QD} / \mathrm{SiO}_{2}$-nanocomposite emission intensity after adding TEOS/ $\mathrm{NH}_{4} \mathrm{OH}$. The sharp decline of QD emission intensity after forming $\mathrm{SiO}_{2}$ encapsulation was reported in several previous studied. This PL-intensity decrease can be demonstrated by the non-radiative recombination channels when the TEOS was added, as the hydrolyzed TOES could induced the damages on QDs' surface during ligand exchange reaction, as shown in Figure 3. Particularly, the partially hydrolyzed TEOS molecules rapidly exchanged the ligands on QDs' surface, leading to the disorganized distribution on QD surface, causing the PL intensity to drop. Besides, the $\mathrm{OH}^{2}$ ions of $\mathrm{NH}_{4} \mathrm{OH}$ solution removed the organic ligands on QD's surface, probably due to the formation of surface hydroxide 
complexes and incomplete passivation. As a result, this contributed to the low intensity of QD-SiO 2 nanocomposite particle. Thus, to enhance the $\mathrm{SiO}_{2}$-encapsulated QD's PL intensity, the $\mathrm{NH}_{4} \mathrm{OH}$ direct reaction should be prevented. Therefore, as $\mathrm{NH}_{4} \mathrm{OH}$ was applied for TEOS hydrolysis in our research, the reduction of PL is unavoidable, see Figure 4.

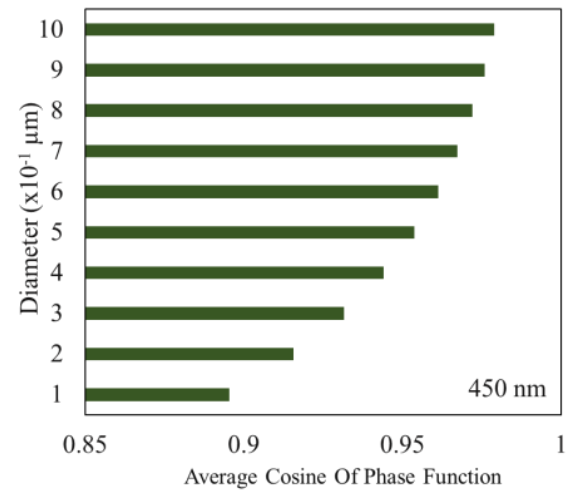

(a)

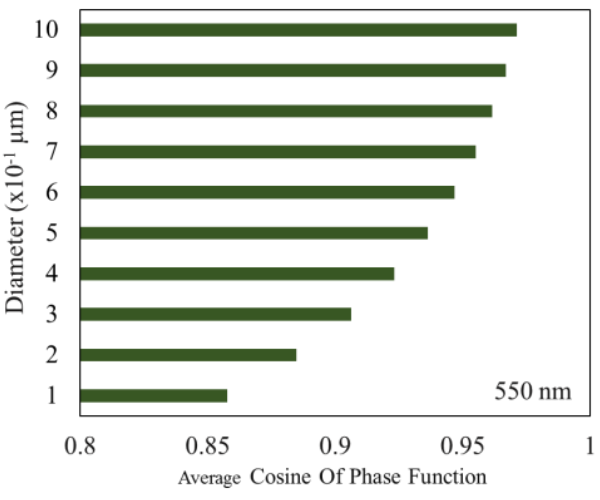

(b)

Figure 3. The phase function of $\mathrm{SiO}_{2}$ particles at, (a) $450 \mathrm{~nm}$, (b) $550 \mathrm{~nm}$

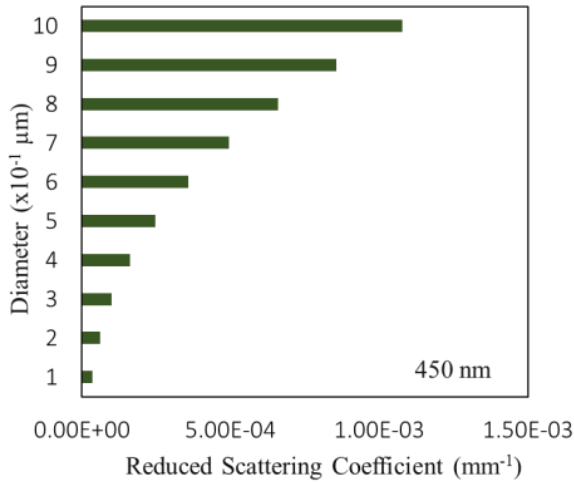

(a)

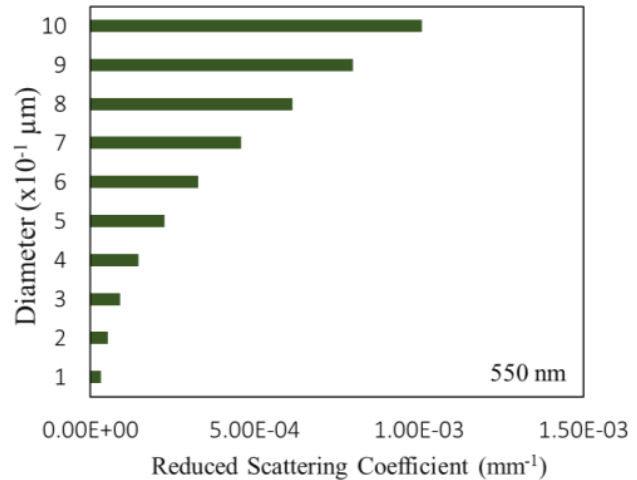

(b)

Figure 4. The reduced scattering coefficient of $\mathrm{SiO}_{2}$ particles at different sizes and wavelength at, (a) $450 \mathrm{~nm}$, (b) $550 \mathrm{~nm}$

In order to limit the reduction in the emission intensity of quantum dots when $\mathrm{SiO}_{2}$ encapsulation is formed, different methods including initial silanization [21] and formation of multiple shells on QDs were proposed. In details, the first silanization method was confirmed to be able to remain the quantum output of $\mathrm{QD}-\mathrm{SiO}_{2}$ nanocomposites as high as that of the original quantum dots. Hence, the initial silanization was adopted in this research for minimizing the deterioration rate in the intensity of QDs' emission after the formation of silica encapsulation. The QDs were silanized by 10-minute stirring the mixture of 3aminopropyl-triethoxysilane (APS) and spray-dispersed QD solution, followed by the addition of TEOS/ $\mathrm{NH}_{4} \mathrm{OH}$ to increase the thickness of $\mathrm{SiO}_{2}$ encapsulating layer. The preservation of the initial QD's was observed after adding APS, see Figure 5. The PL intensity showed a very small reduction with the use of TEOS/ $\mathrm{NH}_{4} \mathrm{OH}$ when being reacted for 1 hour. As the reaction time became longer, the PL reduction was at about $15-20 \%$, however, this rate is much lower than that of the APS-free synthesis. In this case, the PL intensity drop could be attributed to the incomplete passivation on QD's surface by APS. 


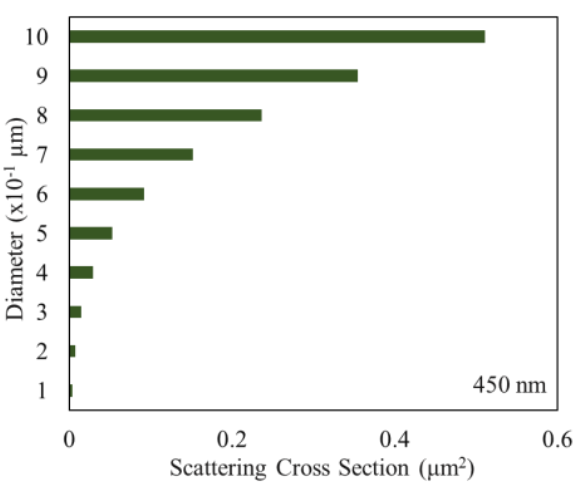

(a)

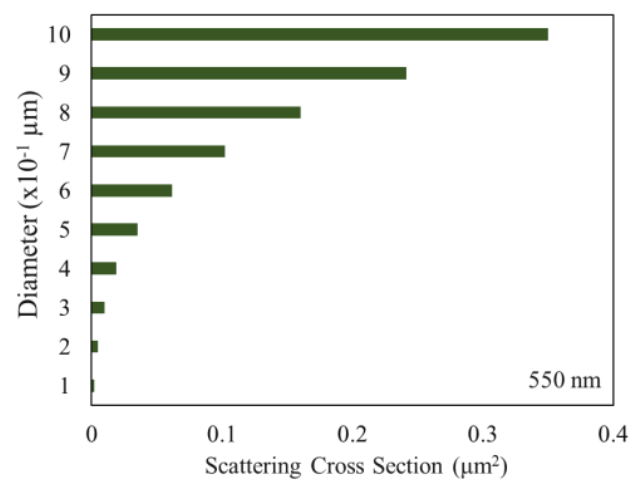

(b)

Figure 5. The scattering cross section of $\mathrm{SiO}_{2}$ particles at different sizes and wavelength at, (a) $450 \mathrm{~nm}$, (b) $550 \mathrm{~nm}$

\section{RESULTS AND DISCUSSION}

The lumen output at various $\mathrm{SiO}_{2}$ particles amounts was calculated and demonstrated in Figure 6. At the starting point of $1 \%$ concentration, the $\mathrm{SiO}_{2}$ configuration has shown $12 \%$ higher light output in comparison to the value of the conventional configuration. The optical properties of the $\mathrm{SiO}_{2}$ particles that improved the extraction of blue light have enabled the yellow light to reach higher intensity and resulted in better luminous flux. The explanation for this phenomenon is that the longer scattering wavelength of blue light making the stimulation of yellow phosphor becomes more likely, which leads to the increased yellow light. The luminous flux in $\mathrm{SiO}_{2}$ configuration is only better in the a certain amount of concentration, as the amount of $\mathrm{SiO}_{2}$ rises, the light output begins to decrease because the emitted lights were trapped in the structure, and the re-absorption happened between the materials. In Figure 7 is the CCT deviation in WLEDs structure corresponding to various $\mathrm{SiO}_{2}$ concentrations. The default correlated color temperature in the simulated model dropped from $5319 \mathrm{~K}$ to lower values as the amount of $\mathrm{SiO}_{2}$ in the structure continued to rise, a result yielded from the increased yellow light in the structure. The luminous flux with power sources from 50 to $500 \mathrm{~mA}$ can be inferred from the integrated sphere model presented in Figure 7. The results have shown that with $1 \%$ of $\mathrm{SiO}_{2}$ particles in the structure, the achieved luminous flux was already higher than the conventional model with any power source. The $\mathrm{SiO}_{2}$ particles are also effective in preventing the Lambertian blue light from breaking out of the structure through the silicone layer, which eventually increases the light output of the structure.

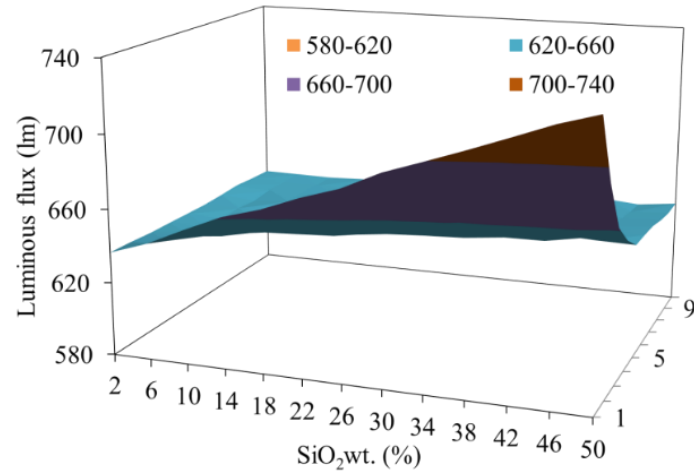

Figure 6. Luminous fluxes of $\mathrm{SiO}_{2}$ particles with different diameters

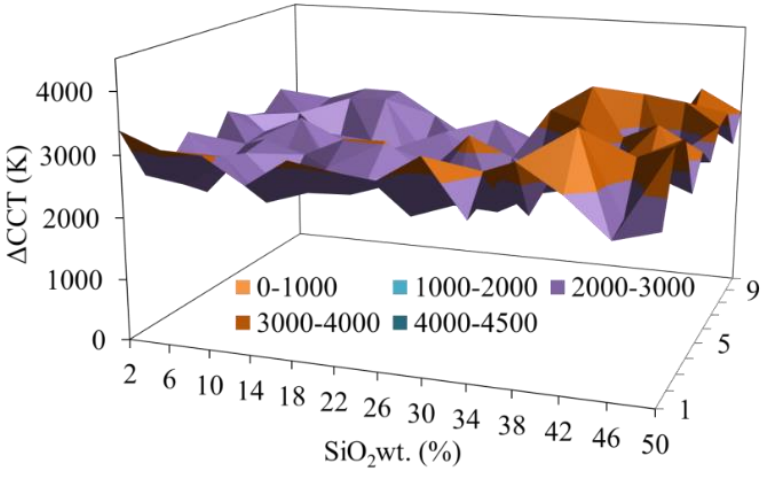

Figure 7. CCT deviations of $\mathrm{SiO}_{2}$ particles with different diameters

The results of angular correlated color temperature in WLEDs packages that were calculated and presented in Figure 7 confirmed the effect of $\mathrm{SiO}_{2}$ nanoparticles in enhancing the color quality of WLEDs. The results of Figure 7 illustrated that the color temperature deviation at different angles decreased once the $\mathrm{SiO}_{2}$ particles were introduced into the structure, and this effect grew stronger as the concentration of $\mathrm{SiO}_{2}$ 
increased. In LEDs configuration without the presence of $\mathrm{SiO}_{2}$, the obtained blue light is at a high amount, which leads to high correlated temperature of $5319 \mathrm{~K}$ in the conventional configuration and low color quality. As the $\mathrm{SiO}_{2}$ particles were doped into the lighting device, the CCT deviation began to improve. The most noticeable improvement was the eradication of color deviation at $0^{\circ}$ and $70^{\circ}$, a result that proved the effect of $\mathrm{SiO}_{2}$ in strengthening the performance lighting configuration. However, to understand further the optical influences of $\mathrm{SiO}_{2}$ layer, experiments regarding the transmission, absorption were conducted. The results when compared to the conventional configuration confirmed that the absorption ability of $\mathrm{SiO}_{2}$ layer is $32 \%$ to $42 \%$ higher at $460 \mathrm{~nm}$ wavelength. This absorption ability in the $\mathrm{SiO}_{2}$ creates more yellow light in the $\mathrm{SiO}_{2}$ structure, which eventually increases the lighting efficiency.

\section{CONCLUSION}

This research examines and proposes an innovative LED structure to achieve better optical performance. The LEDs we suggested utilized the $\mathrm{SiO}_{2} \mathrm{NPs}$ in nano-pillars and an empty space attached to the GaN layer in the PSS inside LEDs. The results from the experiment showed the lumen output increased by $13.5 \%$ in accordance to IQE enhancement. The simulation suggested that nano-pillar on GaN template helps to trap the upwardly excited photons, and get the crowded photons scattered, which is beneficial to the enhancement of the light extraction efficiency of the LED package.

\section{REFERENCES}

[1] A. K. Dubey, M. Gupta, V. Kumar, and D. S. Mehta, "Laser-line-driven phosphor-converted extended white light source with uniform illumination," Applied Optics, vol. 58, no. 9, pp. 2402-2407, 2019, doi: 10.1364/AO.58.002402

[2] N. T. Canh, T. T. T. Can, and W.-S. Choi, "Electrohydrodynamic jet-sprayed quantum dots for solution-processed light-emitting-diodes," Optical Materials Express, vol. 8, no. 12, pp. 3738-3747, 2018, doi: 10.1364/OME.8.003738.

[3] B. Li, Annadurai, L. Sun, J. Liang, S. Wang, Q. Sun, and X. Huang, "High-efficiency cubic-phased blue-emitting $\mathrm{Ba}_{3} \mathrm{Lu}_{2} \mathrm{~B}_{6} \mathrm{O}_{15}: \mathrm{Ce}^{3+}$ phosphors for ultraviolet-excited white-light-emitting diodes," Optics Letters, vol. 43, no. 20, pp. 5138-5141, 2018, doi: 10.1364/OL.43.005138.

[4] J. Wang, Y. Zhou, and Y. Yang, "Rapid 3D measurement technique for colorful objects employing RGB color light projection," Applied Optics, vol. 59, no. 7, pp. 1907-1915, 2020, doi: 10.1364/AO.382302.

[5] Y. Li, Y. Wang, E. Y. B. Pun, and H. Lin, "Bead-on-string fibers electrospun from terbium acetylacetonate hydrate doped poly methyl methacrylate," Optical Materials Express, vol. 8, no. 2, pp. 276-288, 2018, doi: 10.1364/OME.8.000276.

[6] P. Zhu, H. Zhu, S. Thapa, and G. C. Adhikari, "Design rules for white light emitters with high light extraction efficiency," Optics Express, vol. 27, no. 16, pp. A1297-A1307, 2019, doi: 10.1364/OE.27.0A1297.

[7] Z. Liu, et al., "Effect of the replacement of $\mathrm{Zn}^{2+}$ with $\mathrm{Mg}^{2+}$ in $\mathrm{Ca}_{14} \mathrm{Zn}_{6} \mathrm{Ga}_{10} \mathrm{O}_{35}: \mathrm{Mn}^{4+}$," Optical Materials Express, vol. 8, no. 9, pp. 2532-2541, 2018, doi: 10.1364/OME.8.002532.

[8] N. Anous, T. Ramadan, M. Abdallah, K. Qaraqe, and D. Khalil, "Impact of blue filtering on effective modulation bandwidth and wide-angle operation in white LED-based VLC systems," OSA Continuum, vol. 1, no. 3, pp. 910929, 2018, doi: 10.1364/OSAC.1.000910.

[9] Aaron Kho and Vivek J. Srinivasan, "Compensating spatially dependent dispersion in visible light OCT," Optics Letters, vol. 44, no. 4, pp. 775-778, 2019, doi: 10.1364/OL.44.000775.

[10] R. Hirayama, H. Nakayama, A. Shiraki, T. Kakue, T. Shimobaba, and T. Ito, "Projection of multiple directional images on a volume structure with refractive surfaces," Optics Express, vol. 27, no. 20, pp. 27637-27648, 2019, doi: 10.1364/OE.27.027637.

[11] T. R. Dastidar and R. Ethirajan, "Whole slide imaging system using deep learning-based automated focusing," Express, vol. 11, no. 1, pp. 480-491, 2020, doi: 10.1364/BOE.379780.

[12] F. Jiang, et al., "Efficient InGaN-based yellow-light-emitting diodes," Photonics Research, vol. 7, no. 2, pp. 144148, 2019, doi: 10.1364/PRJ.7.000144.

[13] Y. P. Chang, et al., "New scheme of LiDAR-embedded smart laser headlight for autonomous vehicles," Optics Express, vol. 27, no. 20, pp. A1481-A1489, 2019, doi: 10.1364/OE.27.0A1481.

[14] Y. J. Park, et al., "Development of high luminous efficacy red-emitting phosphor-in-glass for high-power LED lighting systems using our original low Tg and Ts glass," Optics Letters, vol. 44, no. 24, pp. 6057-6060, 2019, doi: 10.1364/OL.44.006057.

[15] X. Huang, J. Liang, B. Li, L. Sun, and J. Lin, "High-efficiency and thermally stable far-red-emitting NaLaMgWO6:Mn4+ phosphorsfor indoor plant growth light-emitting diodes," Optics Letters, vol. 43, no. 14, pp. 3305-3308, 2018, doi: 10.1364/OL.43.003305.

[16] X. P. Li, et al., "Highly stable and tunable white luminescence from Ag-Eu3+co-doped fluoroborate glass phosphors combined with violet LED," Optics Express, vol. 26, no. 2, pp. 1870-1881, 2018, doi: 10.1364/OE.26.001870. 
[17] X. Li, B. Hussain, L. Wang, J. Jiang and C. P. Yue, "Design of a 2.2-mW 24-Mb/s CMOS VLC Receiver SoC With Ambient Light Rejection and Post-Equalization for Li-Fi Applications," in Journal of Lightwave Technology, vol. 36, no. 12, pp. 2366-2375, 15 June15, 2018, doi: 10.1109/JLT.2018.2813302.

[18] Z. Zhou, G. Liu, J. Ni, W. Liu, and Q. Liu, "White light obtainment via tricolor luminescent centers and energy transfer in $\mathrm{Ca}_{3} \mathrm{ZrSi}_{2} \mathrm{O}$ : $\mathrm{Eu}^{3+}, \mathrm{Bi}^{3+}, \mathrm{Tb}^{3+}$ phosphors," Optical Materials Express, vol. 8, no. 11, pp. 3526-3542, 2018, doi: 10.1364/OME.8.003526.

[19] B. Wang, D. S. Li, L. F. Shen, E. Y. B. Pun, and H. Lin, "Eu ${ }^{3+}$ doped high-brightness fluorophosphate laser-driven glass phosphors," Optical Materials Express, vol. 9, no. 4, pp. 1749-1762, 2019, doi: 10.1364/OME.9.001749.

[20] P. C. Grant, et al., "UHV-CVD growth of high quality GeSn using SnCl4: from material growth development to prototype devices," Optical Materials Express, vol. 9, no. 8, pp. 3277-3291, 2019, doi: 10.1364/OME.9.003277.

[21] Y. Li, et al., "395 nm GaN-based near-ultraviolet light-emitting diodes on $\mathrm{Si}$ substrates with a high wall-plug efficiency of 52.0\%@350 mA," Optics Express, vol. 27, no. 5, pp. 7447-7457, 2019, doi: 10.1364/OE.27.007447.

[22] Kyoji Matsushima and Noriaki Sonobe, "Full-color digitized holography for large-scale holographic 3D imaging of physical and nonphysical objects," Applied Optics, vol. 57, no. 1, pp. A150-A156, 2018, doi: 10.1364/AO.57.00A150.

[23] H. Lee, et al., "Color-tunable organic light-emitting diodes with vertically stacked blue, green, and red colors for lighting and display applications," Optics Express, vol. 26, no. 14, pp. 18351-18361, 2018, doi: 10.1364/OE.26.018351.

[24] J. Li, et al., "Double optical gating for generating high flux isolated attosecond pulses in the soft X-ray regime," Optics Express, vol. 27, no. 21, pp. 30280-30286, 2019, doi: 10.1364/OE.27.030280.

[25] S. Kumar, M. Mahadevappa, and P. K. Dutta, "Extended light-source-based lensless microscopy using constrained and regularized reconstruction," Applied Optics, vol. 58, no. 3, pp. 509-516, 2019, doi: 10.1364/AO.58.000509.

[26] J. Lui, A. M. Vegni, L. Colace, A. Neri, and C. Menon, "Preliminary design and characterization of a low-cost and low-power visible light positioning system," Applied Optics, vol. 58, no. 26, pp. 7181-7188, 2019, doi: 10.1364/AO.58.007181. 